\title{
Microscopic modeling of direct pre-equilibrium emission from neutron induced reactions on even and odd actinides
}

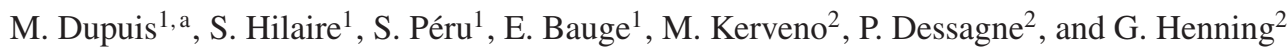 \\ ${ }^{1}$ CEA, DAM, DIF, Arpajon, France \\ ${ }^{2}$ Université de Strasbourg, CNRS, IPHC UMR 7178, 67000 Strasbourg, France
}

\begin{abstract}
Direct inelastic scattering to discrete excitations and pre-equilibrium emission are described within a microscopic model. Nuclear structure information are obtained in the (Quasi) Random Phase Approximation ( (Q)RPA) framework implemented with the Gogny force. The relevant optical and transition potentials are build considering the JLM folding model. Various successful applications are shown for (n,n), (n,n'), $(\mathrm{n}, x \mathrm{n})$ and $(\mathrm{n}, x \mathrm{n} \gamma)$ reactions for spherical and axially deformed even-even or odd targets. The rearrangement corrections to transition potentials and the contribution of unnatural parity excitations to pre-equilibrium emission are discussed. Our model predictions for $\left(\mathrm{n}, \mathrm{n}^{\prime} \gamma\right)$ reactions, for intra- and inter-band transitions in ${ }^{238} \mathrm{U}$, and for the ${ }^{239} \mathrm{Pu}(\mathrm{n}, 2 \mathrm{n})$ cross section are analyzed.
\end{abstract}

\section{Introduction}

Energy production, nuclear waste management, isotope production for medicine, modeling of nucleo-synthesis, all require a precise knowledge of cross sections for various light incoming and outgoing particles for target nuclei spanning the whole mass table. Focusing on nucleon induced reaction below $200 \mathrm{MeV}$, one needs to consider direct reactions, that gather shape elastic and inelastic scattering to discrete states, compound nucleus reactions, that involve particle evaporation after an equilibration stage, pre-equilibrium processes, close from direct reactions but dedicated to inelastic scattering to the continuum where the target level density is large, and finally eventually fission.

We report here recent progresses we made in the modeling of direct and pre-equilibrium reactions starting from a microscopic description of target excitations. We focus on the description of $(\mathrm{n}, x \mathrm{n})$ and $(\mathrm{n}, x \mathrm{n} \gamma)$ reactions for actinides which are of particular interest in nuclear energy applications. New cross section measurements of the $\left(n, n^{\prime} \gamma\right)$ reactions [1-4] provide new challenges to the modeling of various reaction mechanisms. Moreover, microscopic modeling of neutron induced reaction on actinides could help in better determining observables which still cannot be well constrained by measurements, such as the ${ }^{238} \mathrm{U}\left(\mathrm{n}, \mathrm{n}^{\prime}\right)$ and ${ }^{239} \mathrm{Pu}(\mathrm{n}, 2 \mathrm{n})$ cross sections.

Our approach was designed to handle neutron induced reactions from a few $\mathrm{MeV}$ to $200 \mathrm{MeV}$ and for a wide range of target masses including actinides. We rely on the JLM folding model [5-8] to define the optical and transition potentials that are needed to describe direct inelastic scattering within the DWBA approximation [9] for spherical nuclei, and the coupled channels formalism [9] for axially deformed nuclei such as actinides. Transition potentials are calculated

a e-mail: marc.dupuis@cea.fr considering rearrangement corrections [10]. Nuclear structure ingredients, namely local matter densities for the nucleus ground state (GS), as well as energy, spin, parity and local transition densities for each target excitations, are calculated within the (Q)RPA model $[11,12]$ implemented with the Gogny D1S interaction [13].

First, we detail the major hypotheses made in our determination of pre-equilibrium neutron emission for neutron induced reactions. To assess the accuracy of our modeling, we demonstrate its ability to describe direct inelastic scattering to discrete excitations in ${ }^{208} \mathrm{~Pb}$, illustrating the impact of rearrangement corrections on the predicted cross sections. Then, we discuss the direct pre-equilibrium calculation for the ${ }^{208} \mathrm{~Pb}(\mathrm{n}, x \mathrm{n})$ reaction, focusing on the contribution of non natural parity excitations to the neutron emission. Results for ${ }^{238} \mathrm{U}(\mathrm{n}, x \mathrm{n})$ are then analyzed, as well as (n,n' $\gamma)$ cross sections for various intra- and inter-band transitions. Our approach is then extended to neutron scattering on odd actinides. Preliminary results are discussed for ${ }^{239} \mathrm{Pu}(\mathrm{n}, x \mathrm{n})$ reactions, and more particularly for the ${ }^{239} \mathrm{Pu}(\mathrm{n}, 2 \mathrm{n})^{238} \mathrm{Pu}$ reaction. Concluding remarks and prospects are finally drawn.

\section{Direct pre-equilibrium emission}

The direct pre-equilibrium emission is approximated considering all direct inelastic scattering processes to onephonon states predicted by the (Q)RPA model, namely:

$$
|N\rangle=\Theta_{N}^{\dagger}|\tilde{0}\rangle,
$$

where $|\tilde{0}\rangle$ is the correlated GS and $\Theta_{N}^{\dagger}$ is the phonon creation operator of a state with an excitation energy $E_{N}$. The (Q)RPA equations are solved in a spherical or cylindrical harmonic oscillator basis. Solutions for spherical nuclei are such as $|N\rangle \equiv\left|N, J^{\Pi} M\right\rangle$ where $J$ is the total angular momentum, $M$ its projection on the quantification axis and $\Pi$ the parity. Solutions for axially 
deformed nuclei are $|N\rangle \equiv\left|K^{\Pi}\right\rangle$, where $K$ is the total angular momentum projection on the symmetry axis in the intrinsic frame. Details about the RPA and QRPA formalisms for spherical and axially deformed targets are provided in $[11,14,15]$ and $[12,16]$, respectively.

The doubly differential cross section associated to the direct inelastic scattering of a neutron with an incident energy $E_{i}$, an outgoing energy in the range $\left[E_{f}-\frac{\Delta}{2}, E_{f}+\frac{\Delta}{2}\right]$, and in the solid angle $\Omega_{f}=\theta_{f}, \phi_{f}$, reads:

$$
\frac{d \sigma\left(\theta_{f}, E_{f}\right)}{d \Omega_{f} d E_{f}}=\frac{1}{2 \Delta} \int_{E_{f}-\frac{\Delta}{2}}^{E_{f}+\frac{\Delta}{2}} d E \sum_{N} g\left(E_{i}-E-E_{N}\right) \frac{d \sigma_{N}\left(\theta_{f}\right)}{d \Omega_{f}} .
$$

The differential cross section $\frac{d \sigma_{N}\left(\theta_{f}\right)}{d \Omega_{f}}$ corresponds to the direct inelastic scattering to a phonon excitation defined in Eq. (1). For scattering on spherical nuclei, this cross section is calculated within the DWBA approximation. For axially deformed targets, a coupled channel formalism, for which the coupling scheme includes the GS rotational band and a rotational band built on an intrinsic excitation, is considered (see [8] for more details). In Eq. (2), the function $g\left(E_{i}-E-E_{N}\right)$ simulates the finite width of the (Q)RPA states that would result from couplings to continuum states and to two particles - two holes states for RPA or four quasi-particles states for QRPA. It is approximated by a Lorentzian function with a width $\Gamma\left(E_{N}\right)$. Details about this prescription are provided in $[8,17]$.

The optical and transition potentials needed to perform the DWBA or coupled channel calculations are obtained within the JLM-Bruyères (parametrisation provided in [7]) folding model considering the relevant (Q)RPA local matter densities.

\section{3. ${ }^{208} \mathrm{~Pb}\left(n, n^{\prime}\right)$ and $(n, x n)$}

The folding model is first applied to the description of direct inelastic scattering to the yrast $3^{-}$and $5^{-}$states in ${ }^{208} \mathrm{~Pb}$. The ground state matter densities and transition densities are calculated within the RPA framework with the Gogny D1S interaction. The inelastic process induces a variation in the target matter density. If a density dependent effective interaction is used to built the transition potential, this variation induces a dynamical correction that needs to be accounted for. Schematically, following the method of Cheon et al. [10], the transition potential reads:

$$
U_{\mathrm{tr}}=\rho_{\mathrm{tr}}^{G S \rightarrow E_{N} J^{\Pi}}\left(V\left(\rho_{G S}\right)+\rho_{G S}\left\{\frac{\partial V(\rho)}{\partial(\rho)}\right\}_{\rho=\rho_{G S}}\right),
$$

where $\rho_{\operatorname{tr}}^{G S \rightarrow E_{N} J^{\Pi}}$ is the transition density. The second term on the r.h.s accounts for rearrangement corrections. This expression was detailed in the context of the JLM folding model in [8]. Calculated direct neutron inelastic scattering cross sections for the excitation of the yrast $3^{-}$and $5^{-}$states in ${ }^{208} \mathrm{~Pb}$ are compared to experimental data in Fig. 1. The calculations that include rearrangement corrections (red full curves) are in very good agreement with the experimental data. Neglecting these corrections (red dash curves) results in an increase of the cross section magnitude of about
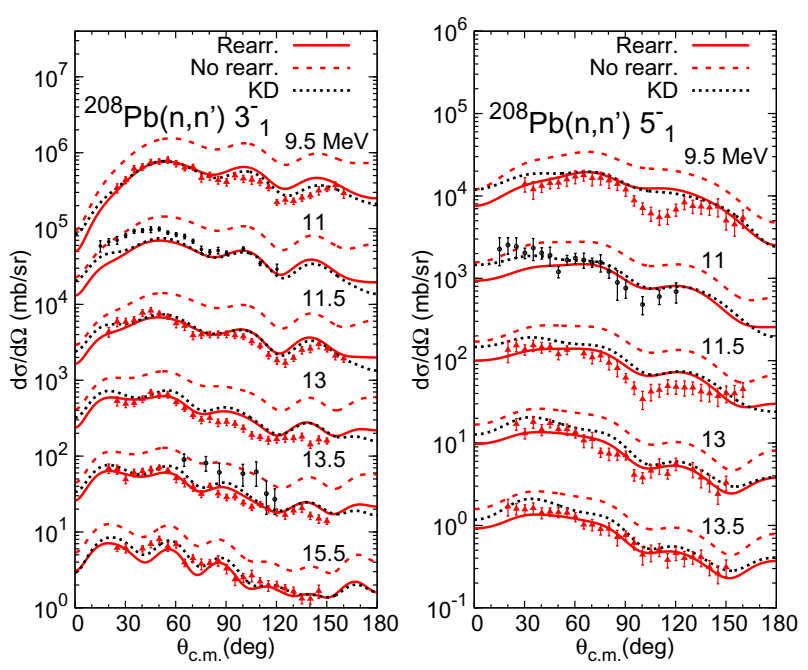

Figure 1. Angular distributions for neutron inelastic scattering on ${ }^{208} \mathrm{~Pb}$ for the yrast $3^{-}$(left panel) and $5^{-}$(right panel) excitations. The red full curves represent JLM calculations, with the RPA transition densities, that include rearrangement corrections. The red dash curves represent the same calculations performed without rearrangement. The black short-dash curves are for collective model calculations (see details in the text). Red symbols represent experimental data from [21]. At 11 and 13.5 MeV, black symbols are for experimental data from [22,23].

100\%. In Fig. 1 are also displayed calculations performed with the collective model [9] (black dotted curves) considering the Koning-Delaroche optical potential [18]. The deformation parameters are calculated following the relation $\beta_{L}=\sqrt{B\left(E_{L}\right)} \frac{4 \pi}{3 Z R_{c}^{L}}$ [9] with $R_{c}=1.2 A^{\frac{1}{3}}$ and the adopted $B\left(E_{L}\right)$ values $0.611(12) 10^{6} \mathrm{e}^{2} \mathrm{fm}^{6}$ [19] for $L=3$ and the experimental value $0.0447(30) 10^{10} \mathrm{e}^{2} \mathrm{fm}^{10}$ [20] for $L=5$. Note that the RPA calculation provides $B\left(E_{L}\right)$ values, namely $0.69210^{6} \mathrm{e}^{2} \mathrm{fm}^{6}$ for $L=3$ and $0.055310^{10} \mathrm{e}^{2} \mathrm{fm}^{10}$ for $L=5$, about $10 \%$ larger. The real, imaginary and spin-orbit part of the optical potentials have been deformed considering the same $\beta_{L}$ for each term. As seen in Fig. 1, the microscopic calculation, including rearrangement, provides results that are close in quality to those obtained with the phenomenological collective model. This agreement is closely related to the characteristic of the two yrast $3^{-}$and $5^{-}$excitations which are surface vibration modes.

We now discuss our model prediction of the preequilibrium contribution to the doubly differential inclusive $(\mathrm{n}, x \mathrm{n})$ cross sections for the $14 \mathrm{MeV}$ neutron induced reactions on ${ }^{208} \mathrm{~Pb}$. Equation (2) is applied considering all natural parity excitations $\left(\Pi=(-)^{J}\right)$, in the excitation energy range $\left[0, E_{\mathrm{in}}\right]$, that are predicted by the RPA model. Individual inelastic scattering cross sections are calculated within the DWBA approximation including rearrangement. The effective two-body interaction used in the JLM model cannot induce unnatural parity transitions $\left(\Pi=-(-)^{J}\right)$. Indeed, those are mostly induced by twobody tensor and spin-orbit interactions which are lacking in the JLM folding model. To evaluate contributions from this process, we performed DWBA calculations determining the transition potentials with the CDM3Y interaction [24] which contains two-body spin-orbit and tensor terms. Our results are compared to data in Fig. 2. 


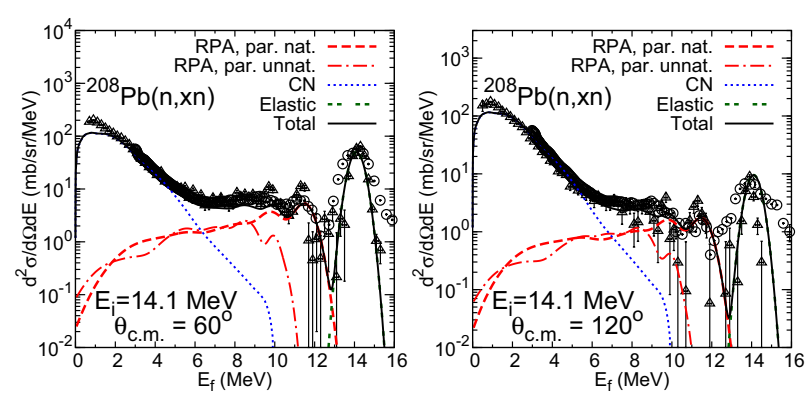

Figure 2. Spectra of neutron emitted at the two outgoing angles $\theta_{c . m}=60$ and $120^{\circ}$ for the $14.1 \mathrm{MeV}{ }^{208} \mathrm{~Pb}(\mathrm{n}, x \mathrm{n})$ reaction. Contributions from various reaction mechanisms (see labels in the plots and details in the text) are summed (black full curves) and compared to two experimental data sets represented as symbols [26,27].
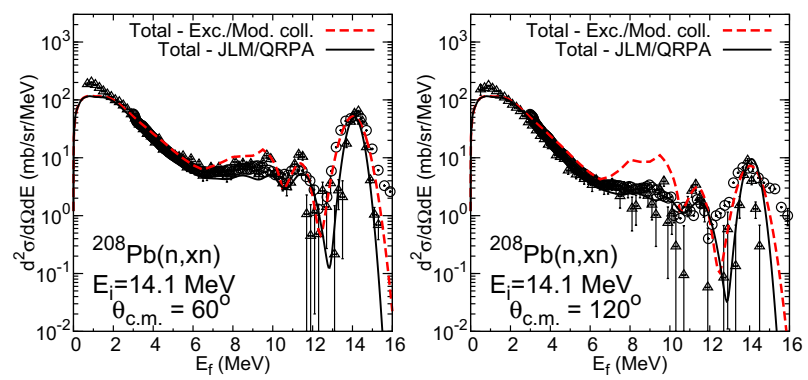

Figure 3. Same reaction and experimental data as in Fig. 2. The red dash curves (black full curves) correspond to the calculation for which the direct and pre-equilibrium contributions are calculated from the exciton and collective models (JLM/QRPA model).

Contributions from various reaction mechanisms, namely compound nucleus evaporation, elastic scattering, and direct inelastic scattering to natural and unnatural parity states, are displayed. The compound nucleus contribution has been calculated with the Talys nuclear reaction code [25]. Our predictions are in fair agreement with the experimental data. On Fig. 3, we compare our model predictions to calculations for which the preequilibrium and direct inelastic scattering to discrete states have been calculated using the two-component exciton model and the collective model, respectively, which are implemented in the Talys code. The collective model that provides differential cross sections for low energy discrete excitations is very well constrained since, for neutron induced reaction on ${ }^{208} \mathrm{~Pb}$, deformations parameters for many states have been determined from previous electron or hadron inelastic scattering measurements. This demonstrates that calculations from our microscopic model can be as accurate as those from well-constrained phenomenological models.

\section{4. ${ }^{238} U(n, x n)$ and (n,n' $\left.\gamma\right)$}

The present modeling of direct pre-equilibrium emission is now applied to neutron induced reactions on actinides. A deformed QRPA calculation provides the target excitations in the intrinsic frame. States in the laboratory frame are obtained considering the projection of one-phonon excitations, Eq. (1) on the total angular momentum. This generates a rotational band for each intrinsic excitation.
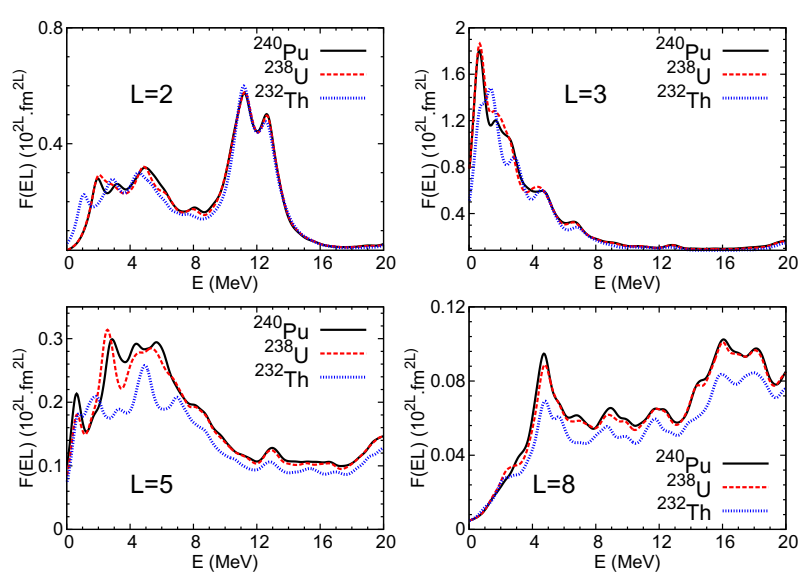

Figure 4. QRPA response functions, Eq. (4), for $L=2,3,5$ and $8 \hbar$ transitions in ${ }^{238} \mathrm{U},{ }^{240} \mathrm{Pu}$ and ${ }^{232} \mathrm{Th}$ as a function of the excitation energy.

States $\left|N J K^{\Pi} M\right\rangle$ of these bands are characterized by the quantum number $J^{\pi} \geq K^{\pi}$ for $K>0, J^{\pi}=$ $0^{+}, 2^{+}, 4^{+} \ldots$ for $K^{\pi}=0^{+}$, and $J^{\pi}=1^{-}, 3^{-}, 5^{-} \ldots$ for $K^{\pi}=0^{-}$(see $[8,12]$ for more details). Direct neutron emission was thus calculated considering the possible excitation of each member of all rotational bands. We considered a spin cut-off of $J=16 \hbar$ which ensures convergence of the calculated cross-section, and provides the probability of creating high spin states which is needed for a precise modeling of (n,n' $\gamma$ ) reactions.

We define the response function for a one body operator $r^{L} Y_{M}^{L}(\Omega)$ as

$$
\begin{aligned}
F(E, L)= & \sum_{N, K \leq J, M} f\left(\Gamma_{N} ; E-E_{N}\right) \mid\left\langle N K J^{\Pi} M\right. \\
& \left.\times\left|r^{L} Y_{M}^{L}(\Omega)\right| G S\right\rangle\left.\right|^{2}
\end{aligned}
$$

with $\Pi=(-)^{L}$ and $J=L$, where $E_{N}$ is the excitation energy, $E, L$ and $\Pi$ are the transferred energy, angular momentum and parity, and $f\left(\Gamma_{N} ; E-E_{N}\right)$ is a Lorentzian form factor with a width $\Gamma_{N}$. These response functions are illustrated in Fig. 4 for the multipolarities $L=2,3,5$ and $8 \hbar$ for the three nuclei ${ }^{238} \mathrm{U},{ }^{240} \mathrm{Pu}$ and ${ }^{232} \mathrm{Th}$. Calculations are performed with $\Gamma_{N}=1 \mathrm{MeV} \forall N$.

Calculated inclusive $(n, x \mathrm{n})$ doubly differential cross sections for a ${ }^{238} \mathrm{U}$ target are depicted in Fig. 5, for the incident energy $9.1 \mathrm{MeV}$ at two outgoing angles. The evaporation (red dash curves) and the neutron fission (blue dash curves) contributions are obtained from the HauserFeshbach model, implemented in the Talys code [25], and the Madland-Nix model [28,29], respectively. Direct elastic and inelastic contributions for the GS rotational band excitations are also calculated within the JLM folding model $[8,30]$ (green double-dash curves). The predicted pre-equilibrium emission (black dash curves), once summed with the contributions from the various reaction mechanisms (black full curves), reproduces the data rather well. As seen in Fig. 4 the QRPA model predicts a strong collective response in the $1-7 \mathrm{MeV}$ excitation energy range. Thus, within this approach, the use of pseudo-states, that were introduced in previous more phenomenological approaches $[31,32]$, is no longer required to describe the high energy part of neutron emission. Here, we have postulated that processes leading 

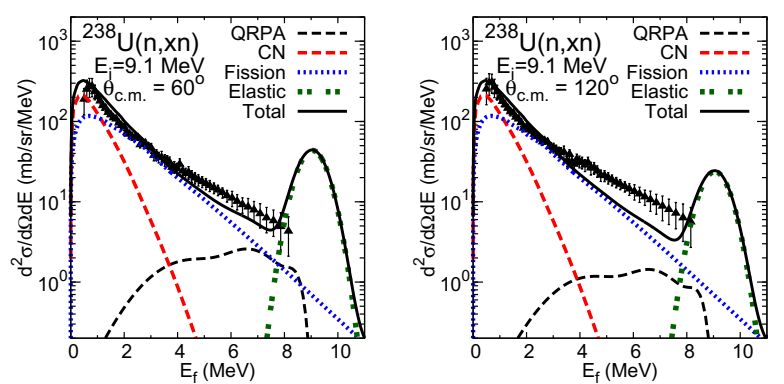

Figure 5. Double differential cross sections for the reaction ${ }^{238} \mathrm{U}(\mathrm{n}, x \mathrm{n})$ at the incident energy $9.1 \mathrm{MeV}$ and the two outgoing angles $60^{\circ}$ (left panel) and $120^{\circ}$ (right panel). The signification of each curve is given in the text. Experimental data [33] are plotted as black symbols.

to the excitation of one-phonon remain dominant with respect to higher order processes, which however may have non negligible contributions for incident energies above about $10-12 \mathrm{MeV}$ for scattering on actinides [8].

Our microscopic modeling of pre-equilibrium emission is then applied to the description of ${ }^{238} \mathrm{U}\left(\mathrm{n}, \mathrm{n}^{\prime} \gamma\right)$ reactions. The neutron emission can occur via a direct inelastic scattering or pre-equilibrium emission process, or via the formation of a compound nucleus followed by a neutron evaporation. We model the direct reaction/pre-equilibrium component of this process using our microscopic model. The spin distribution of the residual nucleus after the emission of one pre-equilibrium neutron is defined by the ratio $\sigma_{J}^{\text {preeq }} / \sigma_{T}^{\text {preeq }}$, with $\sum_{J} \sigma_{J}^{\text {preeq }}=\sigma_{T}^{\text {preeq }}$ where $\sigma_{J}^{\text {preeq }}$ corresponds to the angular and energy integrated cross section defined in Eq. (2) where the sum is limited to the final target excitations of total angular momentum J. If the pre-equilibrium emission is described within the exciton model, the spin distribution of the residual nucleus is not defined and one usually relies on the ad hoc prescription $R_{n}(J)=\frac{2 J+1}{\sqrt{\pi} n^{3 / 2} C^{3}} e^{-\frac{(2 J+1)^{2}}{n C^{2}}}$ with the spin cut-off parameter $C=\sqrt{0.24 n A^{2 / 3}}$ where $n$ is the exciton number of the residual nucleus. Spin distributions calculated with this prescription are compared in Fig. 6 to those stemming from the present JLM/QRPA approach for various incident energies. Our microscopic approach predicts asymmetric distributions that are centered at $J=3-4 \hbar$ while the prescription using the $R_{n}(J)$ functions (prescription implemented in the Talys code) provides distributions centered at $J=7-8 \hbar$. Moreover, microscopic calculations show a significant spreading of the distribution with increasing incident energy while this spreading remains almost constant in the Talys prescription.

As shown in Dupuis et al. [8], the spin distribution of the residual nucleus predicted by the present microscopic pre-equilibrium model allows to reproduce the correct (n,n' $\gamma$ ) cross section magnitude for transitions within the GS rotational band starting from high spin states. This result is illustrated in Fig. 7 for the two $10^{+} \rightarrow$ $8^{+}$and $6^{+} \rightarrow 4^{+}$transitions. We considered three approaches which involve various pre-equilibrium models and various prescriptions of the gamma-ray strength functions. These functions enter the definition of the gamma-ray transmission coefficients which are used in the Hauser-Feshbach model to determine the competition of

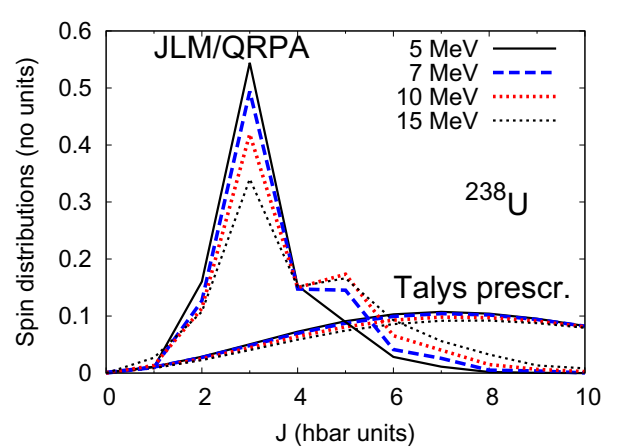

Figure 6. Spin distributions of the residual nucleus formed after the pre-equilibrium emission of one neutron for the $n+{ }^{238} U$ reaction. Distributions obtained with the present JLM/QRPA model are compared to those from the default model used in the Talys code (see details in the text) at various incident energies (see details in the plot).

photon emission with other particles, and the probability of feeding one discrete level from states belonging to the continuous description of target excitations (which starts at $1.2 \mathrm{MeV}$ in our modeling). The first calculation (blue dash curves) was performed using the exciton model for pre-equilibrium emission and the spin distribution defined by the functions $R_{n}(J)$. For this first calculation, the E1 $\gamma$-strength functions were those parametrized by Kopecky [34], and the M1 $\gamma$-strength functions were approximated by a standard Lorentzian form (see p. 60-61 of Ref. [35]). The second calculation (black dotted-dash curves) also uses the exciton model, and considers E1 and M1 $\gamma$-strength functions calculated from the QRPA model $[36,37]$. The third one (red full curves) considers both pre-equilibrium emission and E1/M1 $\gamma$ strength functions calculated from the QRPA model. When QRPA excitations are considered, the magnitude of the $10^{+} \rightarrow 8^{+}$transition is strongly reduced, following the diminution of high spin states production in the n, n' process (see Fig. 6). The $6^{+} \rightarrow 4^{+}$transition magnitude is only slightly reduced. Note that the QRPA model better reproduces the cross section "plateau" observed above $9 \mathrm{MeV}$. For these transitions, varying the $\gamma$-strength functions only weakly affects cross sections magnitudes.

We apply the same approach to describe transitions from states belonging to the first excited band to states member of the GS band. Transitions from the first $1^{-}, 3^{-}$and $5^{-}$states are considered. Results from the three methods detailed above are compared in Fig. 8 to available experimental data including a new dataset recently acquired [2]. Using the QRPA gammaray strength functions increases significantly all the cross section magnitudes. On the contrary, using the JLM/QRPA model for pre-equilibrium reduces the cross sections. Finally, the agreement with the experimental data seemed improved when the QRPA is considered for both pre-equilibrium and gamma-ray strength functions for transitions from the $1^{-}$and $5^{-}$, while the experimental shape and magnitude for transitions from the $3^{-}$state could not be reproduced. A more detailed analysis of these effects is required as other aspects of the modeling, such as the selected level density model, the connection between the discrete and continuous description of the ${ }^{238} \mathrm{U}$ spectrum, could also strongly affect the calculated cross sections. 

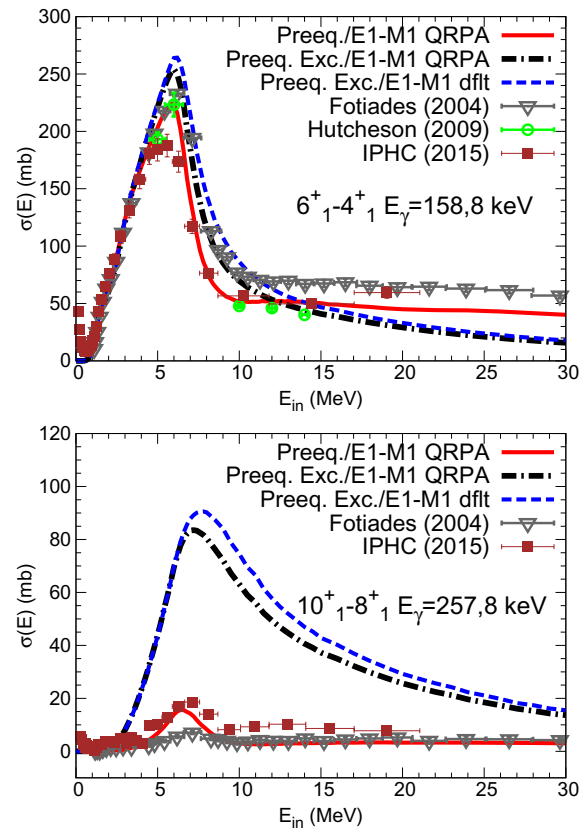

Figure 7. ${ }^{238} \mathrm{U}$ (n,n' $\gamma$ ) cross-sections for the two transitions $6^{+} \rightarrow 4^{+}$(upper panel) and $10^{+} \rightarrow 8^{+}$(lower panel) in the GS rotational band. Experimental data (symbols are defined in the plots - references: IPHC [2], Hutcheson [38], Fotiades [39]) are compared with various Talys calculations (curves, see details in text).

\section{5. ${ }^{239} \mathrm{Pu}(\mathrm{n}, \mathrm{xn})$ and $(\mathrm{n}, 2 \mathrm{n})$}

The description of pre-equilibrium emission for eveneven actinides is extended to scattering on odd actinides, considering the weak coupling approximation: excitations in ${ }^{239} \mathrm{Pu}$ are approximated by the states formed by the couplings of ${ }^{240} \mathrm{Pu}$ target excitations, represented by one phonon QRPA excitations, with the $\frac{1}{2}^{+}$proton hole state. As the cross section magnitude for direct emission, with an angular momentum transfer $L$ and an energy transfer $E$, qualitatively follows the behavior of the response function $F_{L}(E)$ defined in Eq. (4), the calculated preequilibrium cross section is not very sensitive to the details of one excitation but to the collective response which varies slowly with the mass number. This is illustrated in Fig. 4 which shows that the response functions for various angular momentum transfers in ${ }^{240} \mathrm{Pu}$ and ${ }^{238} \mathrm{U}$ are almost identical. This justifies the use of this weak coupling approximation for the calculation of direct preequilibrium emission.

We display in Fig. 9 the double differential cross sections for the reaction ${ }^{239} \mathrm{Pu}(\mathrm{n}, x \mathrm{n})$ at the incident energy 14.1 MeV and the two outgoing angles $60^{\circ}$ and $120^{\circ}$. Neutron evaporation and neutron emission from the fission fragments are calculated following the method described above in the ${ }^{238} \mathrm{U}$ case. As for the ${ }^{238} \mathrm{U}(\mathrm{n}, x \mathrm{n})$, the doubly differential cross section, especially at high emission energy, is rather well described. However, the contribution of non-natural parity excitation are neglected within the JLM+QRPA approach, while they have been proven to be sizable as illustrated in Fig. 2 for the ${ }^{208} \mathrm{~Pb}(\mathrm{n}, x \mathrm{n})$ reaction. To complete our description of the direct neutron emission, we have simulated this contribution by half of the pre-equilibrium cross section predicted within the exciton model. Results corresponding to this prescription
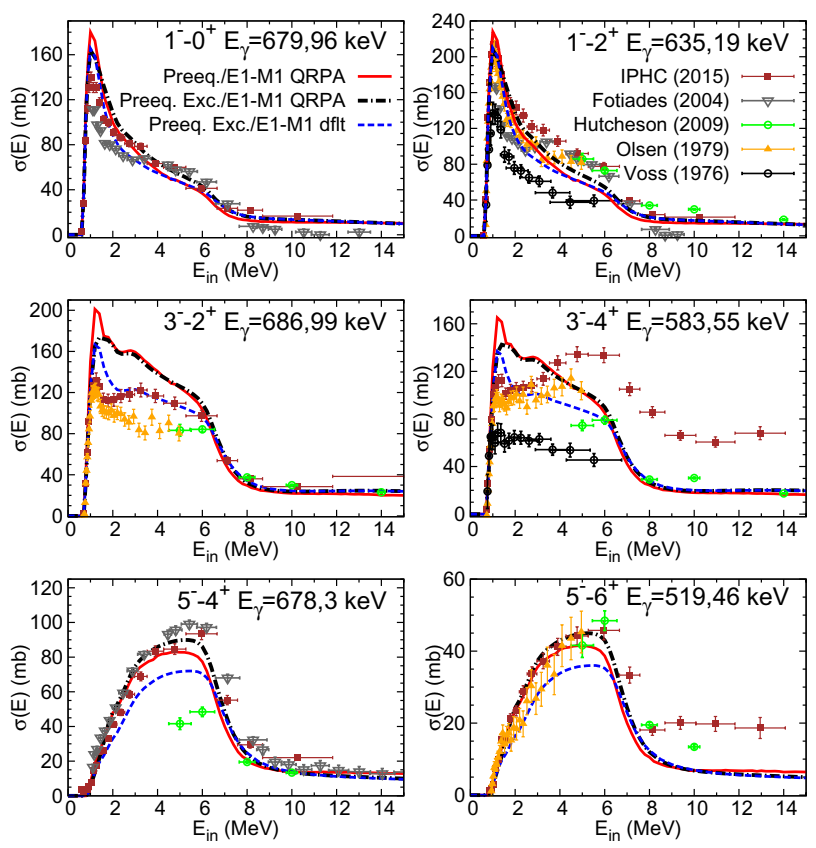

Figure 8. ${ }^{238} \mathrm{U}(\mathrm{n}, \mathrm{n} \gamma \gamma)$ cross-sections for six transitions from states belonging to the first excited band, with $K^{\Pi}=0^{-}$(see Sect. 2), to members of the GS rotational band (see details on each plot). Experimental data (symbols are defined in the second plot - references: IPHC [2], Hutcheson [38], Fotiades [39], Olsen [40], Voss [41]) are compared to various Talys calculations (curves, see details in the first plot and in the text).
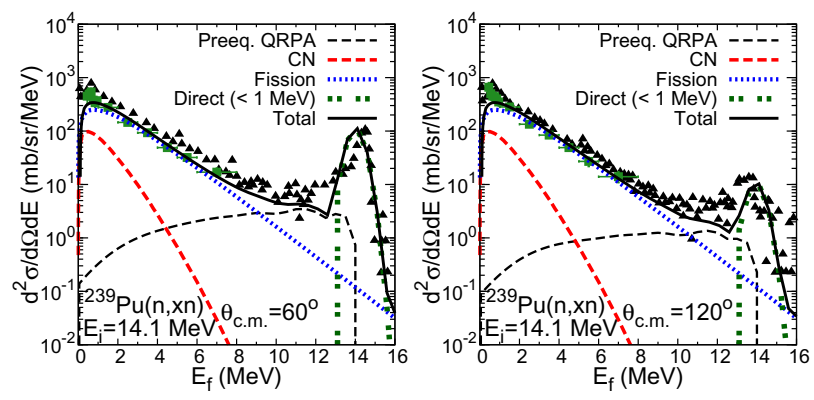

Figure 9. Double differential cross sections for the reaction ${ }^{239} \mathrm{Pu}(\mathrm{n}, x \mathrm{n})$ at the incident energy $14.1 \mathrm{MeV}$ and the two outgoing angles $60^{\circ}$ (left panel) and $120^{\circ}$ (right panel). Calculations (see details in text) are compared to experimental data (black triangles [42], green squares [43]).

are illustrated in Fig. 9 for the inclusive ${ }^{239} \mathrm{Pu}(\mathrm{n}, x \mathrm{n})$ double differential cross sections.

Finally we display in Fig. 10 the predicted ${ }^{239} \mathrm{Pu}(\mathrm{n}, 2 \mathrm{n})$ cross section considering our microscopic modeling of the first direct neutron emission (red full curve). The same calculation obtained with the exciton model is compared (black full curve). The calculation considering the sum of the QRPA excitations and the estimation of non-natural parity excitation approximated by half of the exciton model contribution is also displayed (red dash curve). We note that using QRPA excitations reduces the $(\mathrm{n}, 2 \mathrm{n})$ cross-section from incident neutron energy in the $7-12 \mathrm{MeV}$ range. However, nor the excitation of strong low energy collective states, nor the modification of the spin distribution noticeably affect the cross section shape 


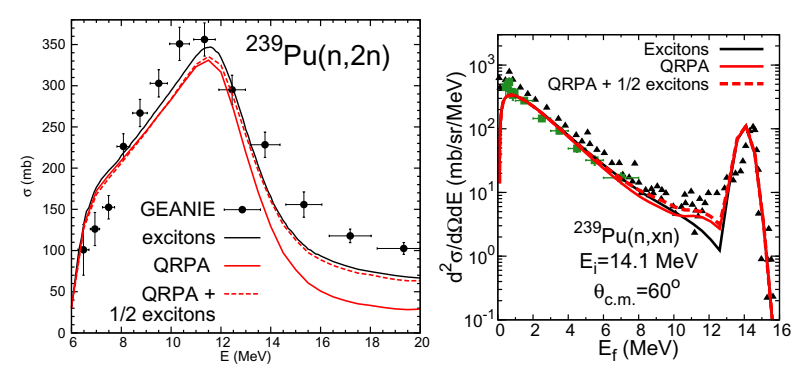

Figure 10. Left panel: ${ }^{239} \mathrm{Pu}(\mathrm{n}, 2 \mathrm{n})$ cross section. Right panel: doubly differential $14.1 \mathrm{MeV}{ }^{239} \mathrm{Pu}(\mathrm{n}, x \mathrm{n})$ cross section at the emission angle $60^{\circ}$. Three calculations corresponding to three different pre-equilibrium models are compared to experimental data $([42,43]$ for $(n, x n)$ and [44] for $(n, 2 n))$. The black full curves correspond to the exciton model, the red full curves to the QRPA model, and the red dash curves to the QRPA model to which half of the exciton contribution has been added.

in the $6-8 \mathrm{MeV}$ region. Our approach could thus not explain the $(n, 2 n)$ cross section behavior in this energy range that was inferred from $(\mathrm{n}, 2 \mathrm{n} \gamma)$ measurements [44].

\section{Conclusions}

Microscopic calculations of neutron direct and preequilibrium emission from spherical and axially deformed nuclei were performed, relying on the JLM folding model and (Q)RPA nuclear structure ingredients calculated with Gogny forces. Specific studies with ${ }^{208} \mathrm{~Pb},{ }^{238} \mathrm{U}$ and ${ }^{239} \mathrm{Pu}$ targets were detailed and showed that our microscopic approach describes various observables with the same accuracy as that of more phenomenological models. Our modeling of direct inelastic neutron scattering to low energy discrete excitations in ${ }^{208} \mathrm{~Pb}$ describes accurately data only if rearrangement corrections are considered. Our model predictions for $(\mathrm{n}, x \mathrm{n})$ doubly differential cross sections reproduce experimental data provided that unnatural parity transitions are accounted for. The agreement with measurements is rather good in the emission energy range that corresponds to an energy transfer to the target of 1 to $5 \mathrm{MeV}$, for which pseudo-states had to be invoked in previous, more phenomenological analyzes. Our model provides spin distributions for the residual nucleus which enables us to properly describe the (n,n' $\gamma$ ) cross section magnitudes for transition within the ground-state rotational bands in ${ }^{238} \mathrm{U}$. Inter-band transitions have been modeled and were shown to be not too sensitive to the spin distribution of the decaying residual nuclei. The (n,n' $\gamma$ ) cross sections sensitivity to the select model for E1 and M1 gamma-ray strength function was characterized. For inter-band transition, using the E1 and M1 $\gamma$-strength functions and the pre-equilibrium model both based on QRPA calculations globally improves the agreement with experimental data. Calculations for the $n+{ }^{239} \mathrm{Pu}$ reaction using the weak coupling approximation were also performed. Our microscopic determination of the direct emission process did not significantly affect the $(n, 2 n)$ cross section shape in the $6-8 \mathrm{MeV}$ incident energy range, but reduces the cross section magnitude in the $8-12 \mathrm{MeV}$ region. The present model is now extended to improve the description of non-natural parity transitions and to evaluate the magnitude of the two-step process contributions, especially in the actinides case. A detailed analysis of the various modeling ingredients for (n,n' $\gamma$ ) reactions is also under progress. The present approach is now extended to $(\mathrm{n}, \mathrm{n}$ ' $\gamma$ ) reactions for $\mathrm{Th}, \mathrm{Zr}$ and $\mathrm{W}$ isotopes to interpret data that were recently measured $[3,4]$.

\section{References}

[1] M. Kerveno et al. Phys. Rev. C 87 (2013)

[2] M. Kerveno et al., Eur. Phys. J. A 51, 167 (2015)

[3] M. Kerveno et al., Proc. of the Int. Conf. on Nucl. Dat. and Tech., Bruges, september 2016, to be published, talk I387

[4] G. Henning et al., Proc. of the Int. Conf. on Nucl. Dat. and Tech., Bruges, september 2016, to be published, talk R277

[5] J.-P. Jeukenne, A. Lejeune, C. Mahaux, Phys. Rev. C 16 (1977)

[6] E. Bauge, J.P. Delaroche, M. Girod, Phys. Rev. C 58 (1998)

[7] E. Bauge, J.P. Delaroche, and M. Girod, Phys. Rev. C 63 (2001)

[8] M. Dupuis, E. Bauge, S. Hilaire, F. Lechaftois, S. Péru, N. Pillet, C. Robin, Eur. Phys. J. A 51(12) (2015)

[9] G.R. Satchler. Direct Nuclear Reactions. (Oxford University Press, Oxford, 1983)

[10] T. Cheon, K. Takayanagi, K. Yazaki, Nucl. Phys. A 437 (1985)

[11] J.P. Blaizot, D. Gogny, Nucl. Phys. A 284, 429 (1977)

[12] S. Péru, G. Gosselin, M. Martini, M. Dupuis, S. Hilaire, J.-C. Devaux, Phys. Rev. C 83 (2011)

[13] J.F. Berger, M. Girod, D. Gogny, Comput. Phys. Commun. 63, 365 (1991)

[14] J.P. Blaizot, D. Gogny, B. Grammaticos, Nucl. Phys. A 265, 315 (1976)

[15] J. Dechargé, L. Šips, Nucl. Phys. A 407, 1 (1983)

[16] S. Péru, H. Goutte, Phys. Rev. C 77 (2008)

[17] M. Dupuis, T. Kawano, J.-P. Delaroche, E. Bauge, Phys. Rev. C 83 (2011)

[18] A.J. Koning, J.-P. Delaroche, Nucl. Phys. A 713, 231 (2003)

[19] T. Kibédi, R.H. Spear, Atomic Data and Nuclear Data Tables 80, 35 (2002)

[20] J. Heisenberg, J. Lichtenstadt, C.N. Papanicolas, J.S. McCarthy, Phys. Rev. C 25, 2292 (1982)

[21] G. Haouat, Private communication, article in preparation.

[22] G.E. Belovickij, L.N. Kolesnikova, I.M. Frank, Yadernaya Fizika 15, (1972)

[23] D.E. Bainum, R.W. Finlay, J. Rapaport, J.D. Carlson, W.G. Love, Phys. Rev. C 16, (1977)

[24] Dao T. Khoa, G.R. Satchler, W. von Oertzen, Phys. Rev. C 56, 954, (1997)

[25] A.J. Koning, S. Hilaire, and M. Duijvestijn. in Nuclear Data for Science and Technology, edited by O. Bersillon, F. Gunsing, E. Bauge, R. Jacqmin, S. Leray (EDP Sciences, Les Ulis, France, 2008), p. 211

[26] T. Elfruth, D. Hermsdorf, H. Kalka, K. Noak, J. Pothig, D. Seeliger, K. Seidel, and S. Unholzer, Technical Report 044, Germ. Dem. Rep. report to the I.N.D.C. (1986)

[27] Y.H.A. Takahashi, E. Ichimura, Tech. Rep. 87, Osaka Univ., OKTAVIAN Reports (1987) 
[28] D.G. Madland, J.R. Nix, Nucl. Sci. Eng. 81, 213 (1982)

[29] A. Chatillon, G. Bélier, T. Granier, B. Laurent, B. Morillon, J. Taieb, R.C. Haight, M. Devlin, R.O. Nelson, S. Noda, J.M. O’Donnell, Phys. Rev. C 89, 014611 (2014)

[30] E. Bauge, J.P. Delaroche, M. Girod, G. Haouat, J. Lachkar, Y. Patin, J. Sigaud, J. Chardine, Phys. Rev. C 61 (2000)

[31] M.B. Chadwick et al., ENDF/B-VII.0: Next Generation Evaluated Nuclear Data Library for Nuclear Science and Technology, Nuclear Data Sheets 107, 2931 (2006)

[32] P. Romain, B. Morillon, H. Duarte, Nuclear Data Sheets 131, 222 (2016)

[33] N.S. Biryukov, B.V. Zhuravlev, N. Kornilov, V.I. Plyaskin, A.P. Rudenko, O.A. Sal'nikov, V.I. Trykova. In 3. All Union conf. on Neutron Phys., Kiev, 9-13 Jun 1975, Vol. 4 (1977)

[34] J. Kopecky, M. Uhl. Phys. Rev. C 41, 1941 (1990)

[35] Talys 1-6 User Manual, http://www.talys.eu/ fileadmin/talys/user/docs/talys1.6.pdf .

[36] M. Martini, S. Péru, S. Hilaire, S. Goriely, F. Lechaftois, Phys. Rev. C 94, 014304 (2016)

[37] S. Goriely, S. Hilaire, S. Péru, M. Martini, I. Deloncle, and F. Lechaftois, Phys. Rev. C 94, 044306 (2016)
[38] A. Hutcheson, C. Angell, J.A. Becker, A.S. Crowell, D. Dashdorj, B. Fallin, N. Fotiades, C.R. Howell, H.J. Karwowski, T. Kawano, J.H. Kelley, E. Kwan, R.A. Macri, R.O. Nelson, R.S. Pedroni, A.P. Tonchev, W. Tornow, Phys. Rev. C 80, 014603 (2009)

[39] N. Fotiades, G.D. Johns, R.O. Nelson, M.B. Chadwick, M. Devlin, M.S. Wilburn, P.G. Young, J.A. Becker, D.E. Archer, L.A. Bernstein, P.E. Garrett, C.A. McGrath, D.P. McNabb, W. Younes, Phys. Rev. C 69, 024601 (2004)

[40] D.K. Olsen, G.L. Morgan, J.W. McConnell, in Proceedings of the International Conference on Nuclear Cross Sections for Technology: Knoxville, TN, 22 Oct. 1979, edited by J.L. Fowler, C.H. Johnson, C.D. Bowman (National Bureau of Standards, Washington, 1979), p. 677

[41] F. Voss, S. Cierjacks, D. Erbe, G. Schmatz, Kernforschungszentrum Karlsruhe Rep. No. 2379, 1976 (unpublished)

[42] J.L. Kammerdiener, Technical Report 51232, U.C., Lawrence Rad. Lab. (Berkeley and Livermore), 1972

[43] J. Voignier, G. Clayeux, F. Bertrand. In 3rd Conf. Neutron Cross-Sections + Tech., Knoxville, Vol. 1, (1971)

[44] L.A. Bernstein et al., Phys. Rev. C 65, 02160 (2002) 